\title{
MULTIOBJECTIVE OPTIMIZATION OF HARD DISK SUSPENSION ASSEMBLIES: PART I-STRUCTURE DESIGN AND SENSITIVITY ANALYSIS
}

\author{
Yee-Pien Yang and Chein-Cherng Tu \\ Department of Mechanical Engineering, National Taiwan University, Taipei, Taiwan 106, \\ Republic of China
}

(Received 23 September 1994)

\begin{abstract}
A multiobjective optimization of the suspension assemblies of hard disk drives is demonstrated. The natural frequencies of the suspension assembly are treated as objective functions subject to side constraints of design variables that describe the shape of the suspension. With the help of an interface program the multiobjective optimizer communicates with an advanced finite element analysis program, without writing a single source code. The gradient information in the optimal design is calculated by finite differences, and the sensitivity analyses are performed numerically with respect to design variables. Preluading and air bearing effects are both considered in the optimal design. Moreover, a final solution is determined by the decision maker with additional knowledge and engineering experience.
\end{abstract}

\section{INTRODUCTION}

High technologies of the magnetic storage devices are being rapidly developed year in and year out. The hard disk drives have been made small and lightweight, with high-area recording density as well as fast and accurate access. Therefore, the slider is required to be kept extremely close to the rotating disk, and the flying height is retained as small as possible for submicron positioning precisions [1]. Some disk drives are already close to head-disk contact with flying heights less than $0.0254 \mu \mathrm{m}$ [2]. Since the flying height is observed always to oscillate but at a very small amplitude [3], its excessive vibration induced by disturbances must cause catastrophic damage to the hard disk system. Major sources of disturbances include vibrations from actuator arm, surface roughness of the disk, spindle/ bearing inaccuracies [4], rotating flow around the suspension $[5,6]$, and so on.

To reduce the magnitude of the flying height fluctuations, either passive or active designs of the suspension assemblies or servo actuators can be used. For servo controls, Hanselmann and Moritz [7] used such a high-bandwidth control of the head-positioning mechanism in a Winchester disk drive that the structural mechanics effects can be neglected. For passive designs, Yamaguchi et al. [5] proposed that the flying height fluctuation can be reduced by an aerofoil-type suspension. Mizoshita et al. [8] pointed out that the resonance of the suspension assembly results in many peaks in the frequency response of the flying height fluctuation, hence a suspension assembly with higher resonant frequencies and sufficient damp- ing will improve the dynamic characteristics of the head slider.

It is quite intuitive and natural for the design objective to raise natural frequencies of the suspension assembly so that it will not be excited easily by undesirable disturbances. There exist two strategies of the structure optimization with frequency requirements. Either the weight of the structure is minimized subject to frequency constraints, or the frequencies are maximized subject to the constraint on the shape, weight or frequency distributions. It is very often that a designer minimizes structural weight with frequency constraints, as shown in Felix and Vanderplaats [9], Chattopadhyay and Walsh [10] and McGee and Phan [11], and was reviewed by Grandhi [12]. In this strategy, scaling procedures are required to adjust frequencies after each iteration to the level specified by the constraints [13]. Therefore, difficulties would arise if the element stiffness matrices are nonlinear in terms of design variables or multiple frequencies are involved.

It is relatively straightforward to treat natural frequencies as objective functions. Structural optimization with multicriterion objectives, which were expressed in terms of displacement, stress, compliance, eigenvalue, or some other measure of structural performance were described in Bendsoe et al. [14]. Szyszkowski [15] presented a method of optimization of maximum frequency of free vibrations, handling structures which might experience multimodal eigensolutions during the solution phase. Szyszkowski and King [16] derived optimality criteria to maximize a set of frequencies for a structure of given weight. An error norm was proposed and used to determine the 
values of design variables and Lagrange multipliers at optimum.

Part I of this paper presents the multiobjective optimization technique and treats natural frequencies as objective functions, subject to some side constraints on the design variables of the suspension assembly. In addition, the sensitivity of objective functions to design variables are analyzed. In Part II we use the integrated structure and control optimization technique, by which the performance index of the linear quadratic regulator is incorporated with frequency objectives.

\section{MODELING OF SUSPENSION ASSEMBLY}

The hard disk suspension assembly consists of a mounting block, suspension beam, flexure and slider. Figure 1 shows an unloaded suspension, which is undeformed prior to loading onto the disk. The suspension is connected with the mounting block by seven weld points. The slider is attached on the flexure with epoxy adhesive in the shaded area, and the flexure is attached to the suspension beam by four

$\dagger$ ABAQUS is a registered trademark of Hibbit, Karlsson and Dorensen, Inc. weld points of diamond shape. The slider and the suspension beam are separated by a hemispherical flexurc knob so that the slider is able to perform pitch and roll motions in its operation mode. The dimensions of each part of the suspension assembly are listed in Appendix A.

The finite element model is created with the undeformed state of suspension. After loading onto the hard disk, the suspension undergoes a preloading force between 0.093 and $0.147 \mathrm{~N}$ [17], and the angle between the suspension and the base line of the mounting block reduces about $2^{\circ}$. This loading process can be performed by analysis procedures of ABAQUS, $\uparrow$ where the user simply divides the loading histories into steps and comes up with the deformed state of pre-stress for subsequent analyses.

The air bearing that separates the slider from the media during the operation is approximated by four linear springs supporting at four corners of the slider. Since the flying height at the front corners of the slider is higher than that at the rear corners, the stiffness of each front corner is modeled by $1 \times 10^{5} \mathrm{Nt} \mathrm{m}^{-1}$, while the stiffness of each rear corner is $1.5 \times 10^{5} \mathrm{Nt} \mathrm{m}^{-1}$. The corresponding flying height is around $0.25 \mu \mathrm{m}$ under a load force about $0.01 \mathrm{~kg}$, according to the previous experiments on the measurement of flying height [18] as shown in Fig. 2.

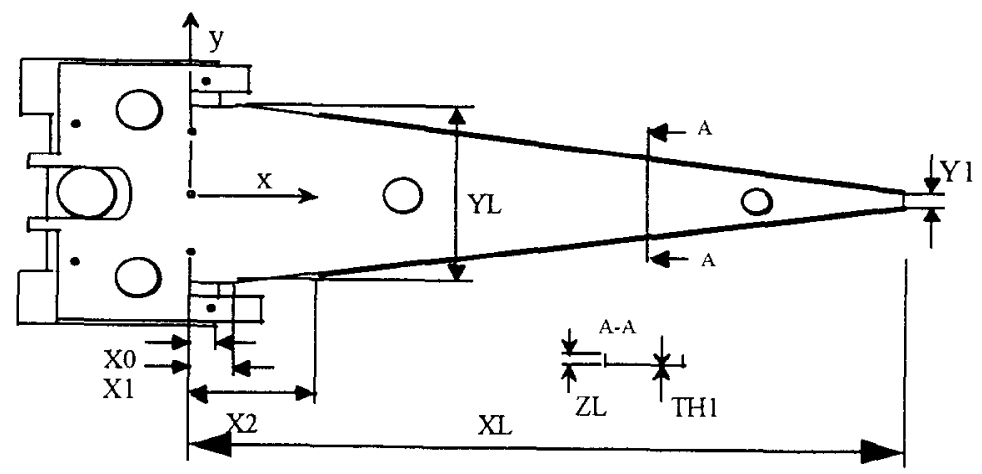

(a)

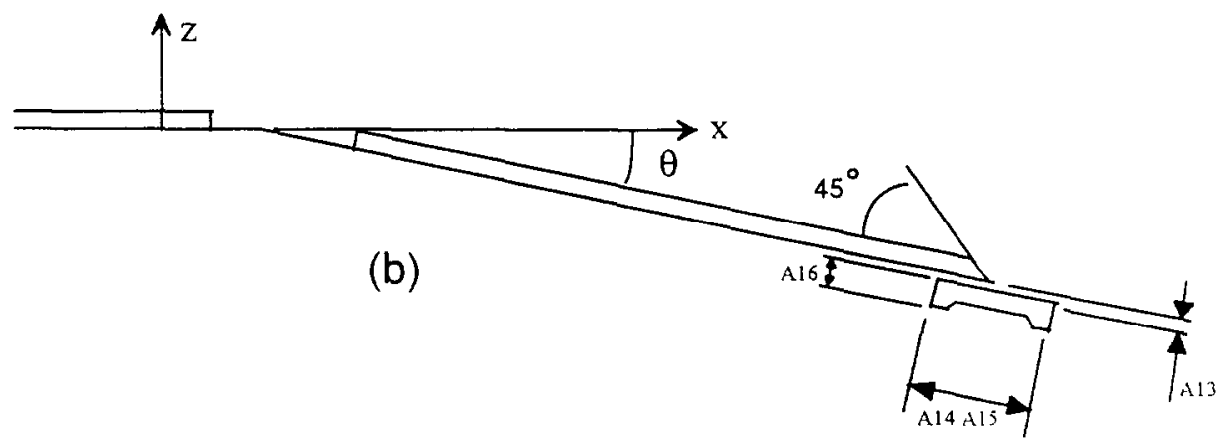

Fig. 1. Suspension assembly dimensions. (a) Top view of suspension beam. (b) Lateral view of suspension beam. (c) Flexure. (d) Slider. (Continued opposite.) 


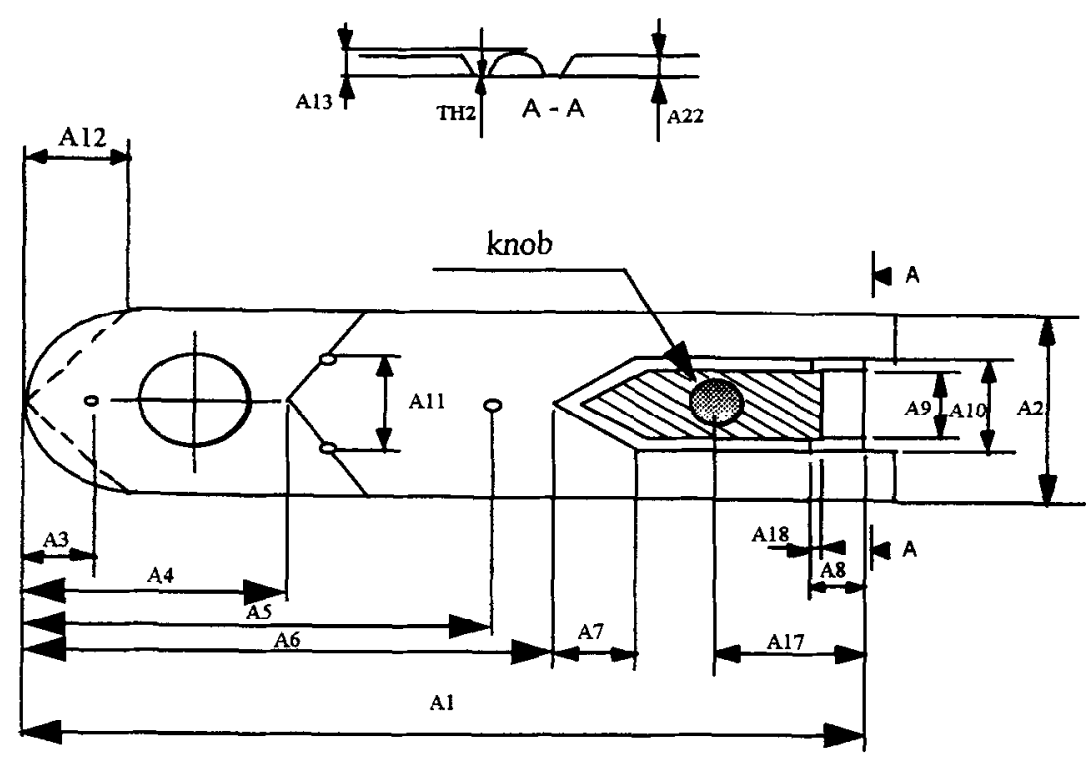

(c)
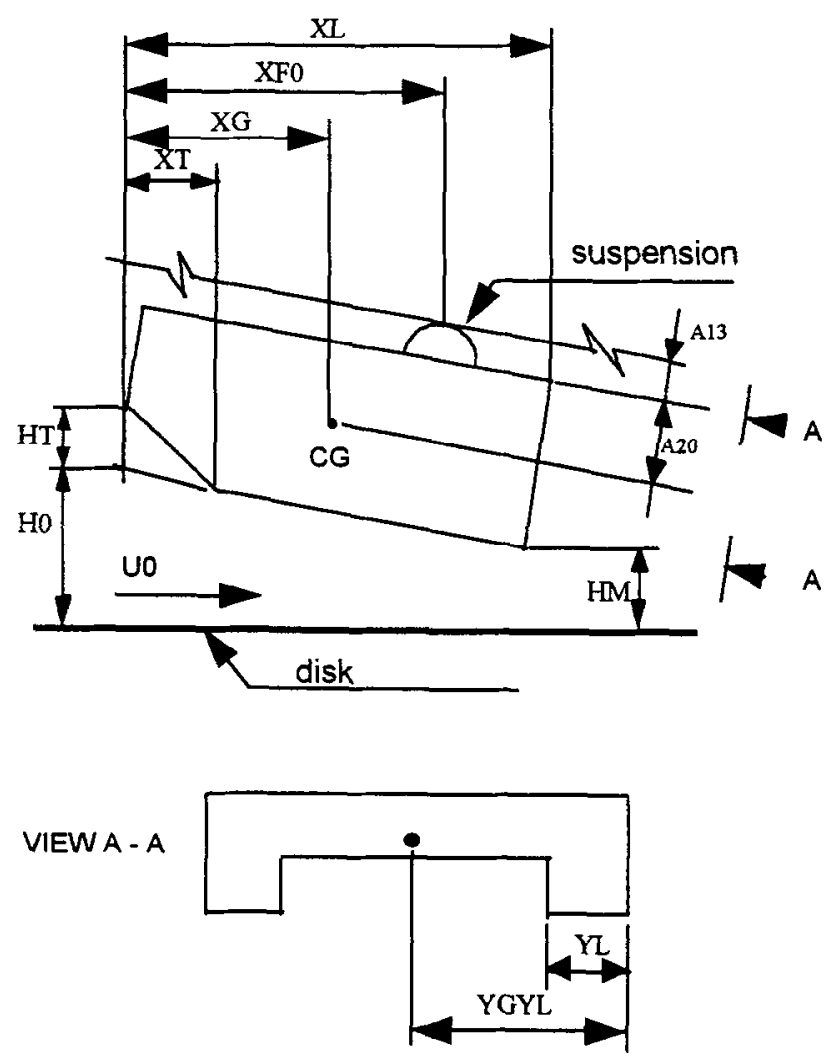

(d)

Fig. 1-Continued. 


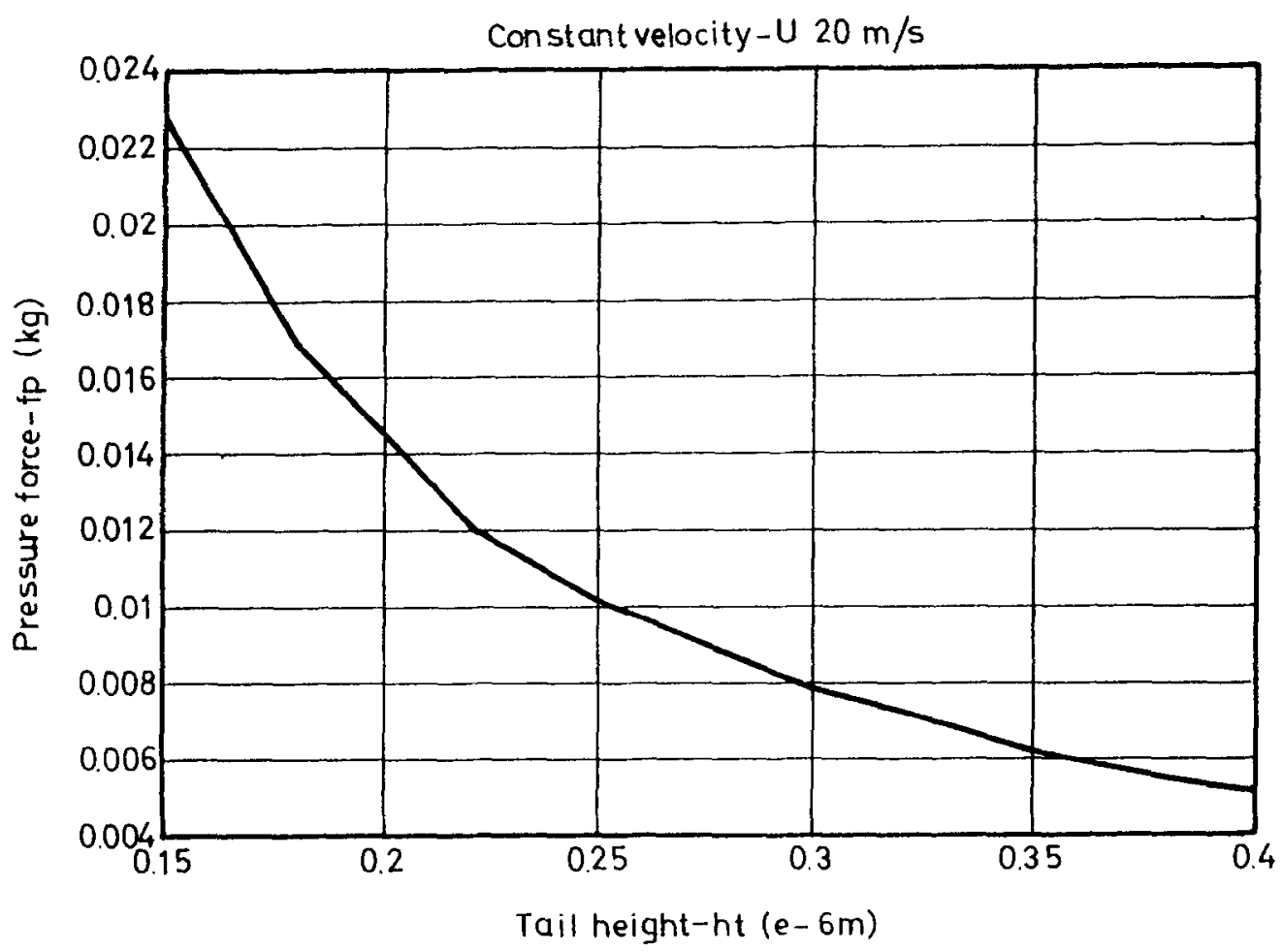

Fig. 2. Resultant air bearing load vs flying height of slider.

\subsection{Finite element model}

The finite element model for the suspension assembly includes a grand total of 232 elements, 321 nodes, with 1794 degrees of freedom (DOF). Two types of elements are used. For the suspension and flexure, we use the quadrilateral thin shell element (S4R5) of four nodes, each of which includes three orthogonal displacements and two rotations along the shell plane. For the slider, the eight-node linear brick $(\mathrm{C} 3 \mathrm{D} 8 \mathrm{H})$ is used, incompressible and hybrid with 3 displacement degrees of freedom for each node. The mounting block is regarded as a fixed rigid body, to which the suspension beam is connected by weld points.

The mesh generation subroutine defines nodal coordinates as functions of design variables. Four shape parameters are selected as design variables:

$y b$-width of the base of the suspension;

$x d$-length of the suspension base attached on the mounting block;

$\times 2--$ distance between the bending corner and the suspension flaps;

$w h$-height of the suspension flaps.

Other parameters are left as constants, including the thickness $(t h)$, tip width $(y d)$ and total length $(l)$ of the suspension, the distance from the edge of the mounting block to the line where the suspension width tapers, and the sizes of flexure and slider. The reasons why we do not choose all the above parameters as design variables are explained in Section 4 with sensitivity analyses. The finite element models of the suspension assembly, as well as its components, are shown in Fig. 3.

As shown in Fig. 1, located at $x=0$ are five nodes of weld points, restrained in all degrees of freedom. At $x=X 0$, modeled are five nodes that are not welded but supported on the mounting block; therefore, three inner nodes are restrained in their three orthogonal directions of displacement, while two other nodes are left free. At the four weld spots between the flexure and the suspension, all their degrees of freedom are compatible. At the contact point pivoting the flexure knob and the suspension, the two nodes that correspond to each component are also constrained so that their vertical displacements are consistent with each other, while rotations are left free.

Since the large deformation in the loading process of the suspension onto the hard disk is a nonlinear problem, ABAQUS uses Newton's method with iterations to come up with the loaded state of the suspension with pre-stress and pre-stain distributions in the finite element model. Figure 4 describes the loading process and pre-stress distribution of the suspension assembly. This model is used in the subsequent dynamical analysis for each optimization loop. 


\subsection{Optimization model and algorithm}

The equations of motion of the suspension assembly for the finite element model of order $N$ have the form

$$
\mathbf{M}(x) \ddot{q}+\mathbf{C}(x) \dot{q}+\mathbf{K}(x) q=\mathbf{B} u,
$$

where $\mathbf{x}$ is a vector of design variables, $\mathbf{M}(x)$ is a positive definite symmetric mass matrix, $\mathbf{K}(x)$ is the nonnegative symmetric stiffness matrix, including the equivalent stiffness due to the preload produced pre-stress and pre-strain, and $q$ is the generalized nodal coordinates. In the modal analysis during the optimization process, the input $u$, its influence matrix $\mathbf{B}$, and the damping matrix $\mathbf{C}(x)$ are neglected while searching for the system's natural frequencies and natural modes. Once we obtain the optimal design of the suspension assembly, the modal damping ratios are included in the modal equations so that more analyses, such as time responses, vibration controls, etc. can be performed.

The first bending and torsional modes frequently occur in the following way: the first mode is a bending mode and the second torsional; or the first mode is a torsional mode and the second bending [1]. Other types of modal sequence rarely happen. However, the third mode may be a bending, torsional or their hybrid mode. Therefore, the objectives of the optimization of suspension assembly are that:

(1) the natural frequencies of the first bending and torsional modes are designed as high as possible so that they arc not casily excitcd by disturbances, such as $60 \mathrm{~Hz}$ disk rotation speed and its harmonics [18], and the air-flow between disks [5];

(2) the second and the third natural frequencies take apart as far as possible. Therefore, it is not
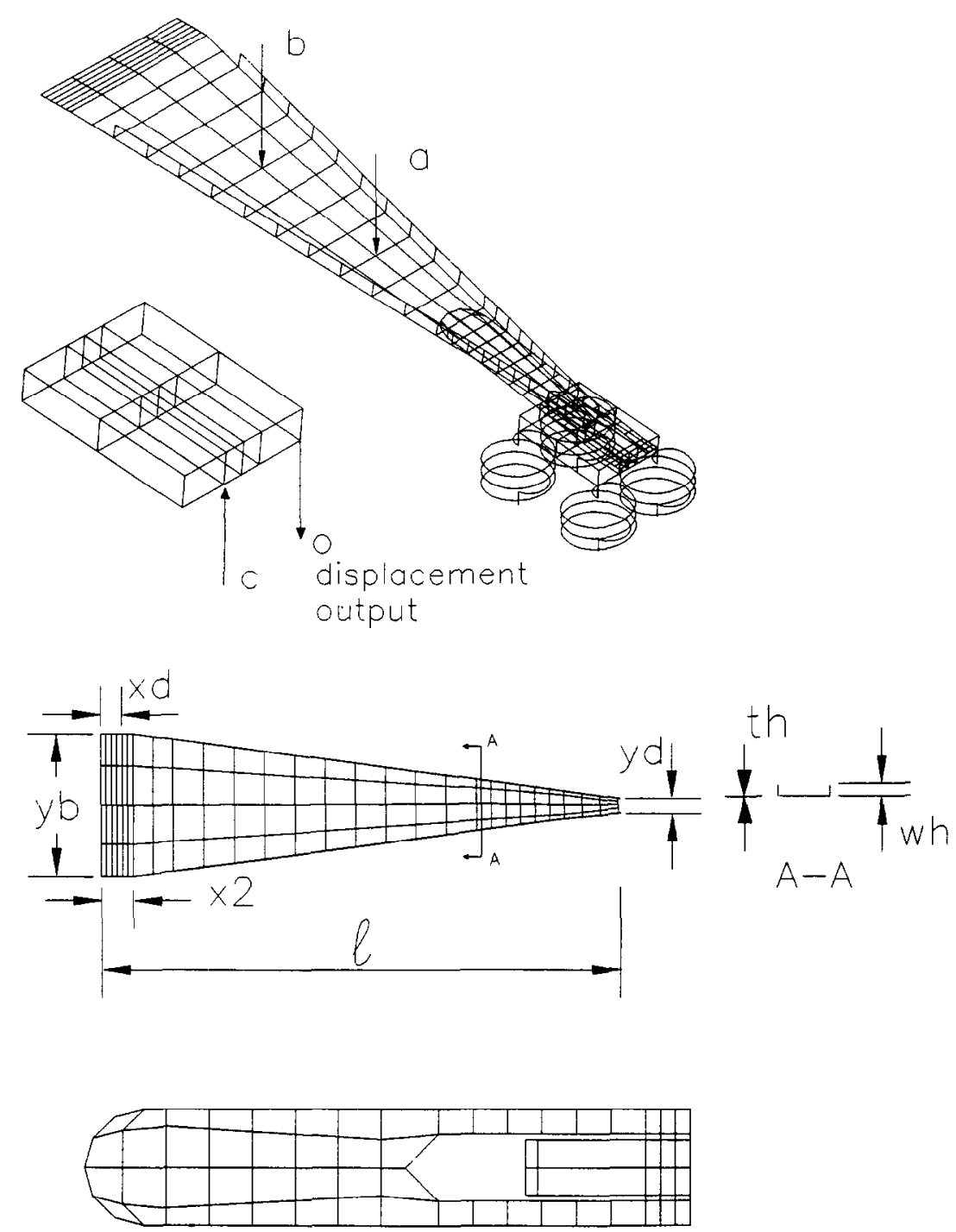

Fig. 3. Finite element model of suspension assembly. 


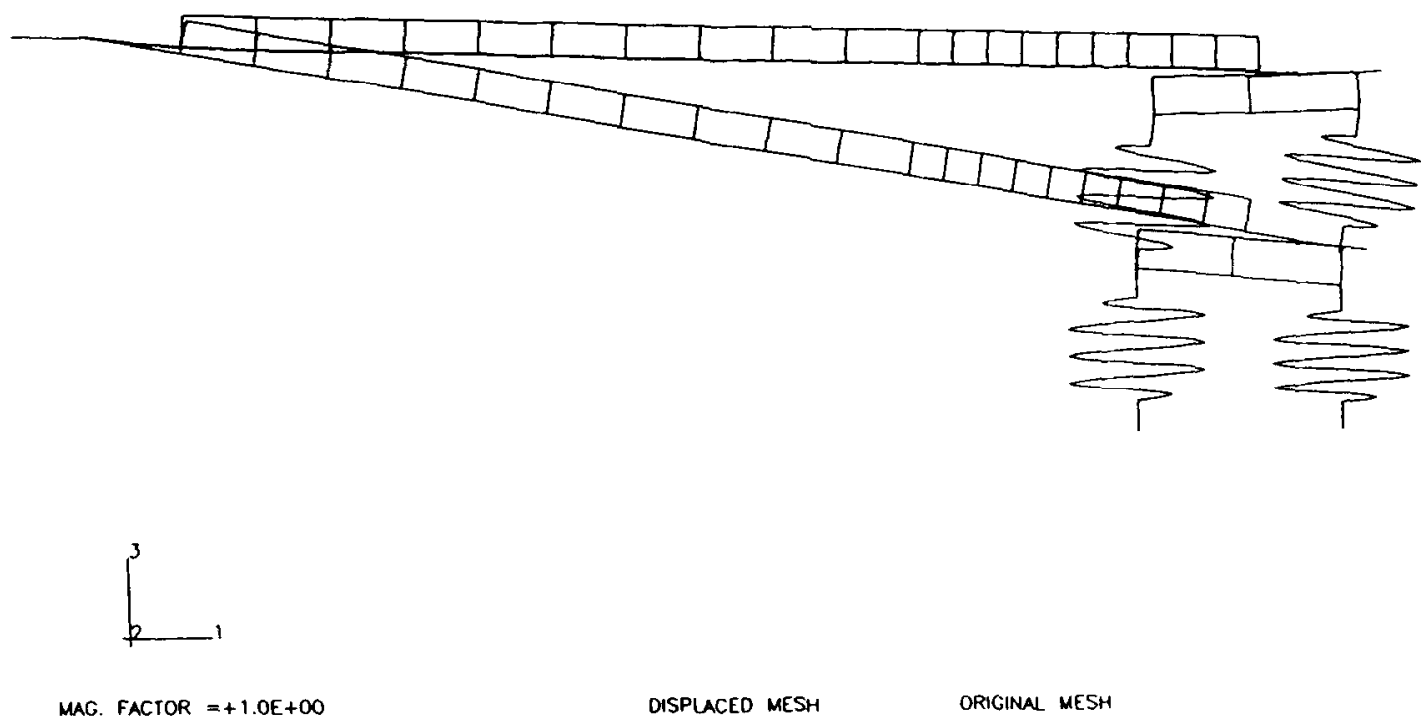

(a)

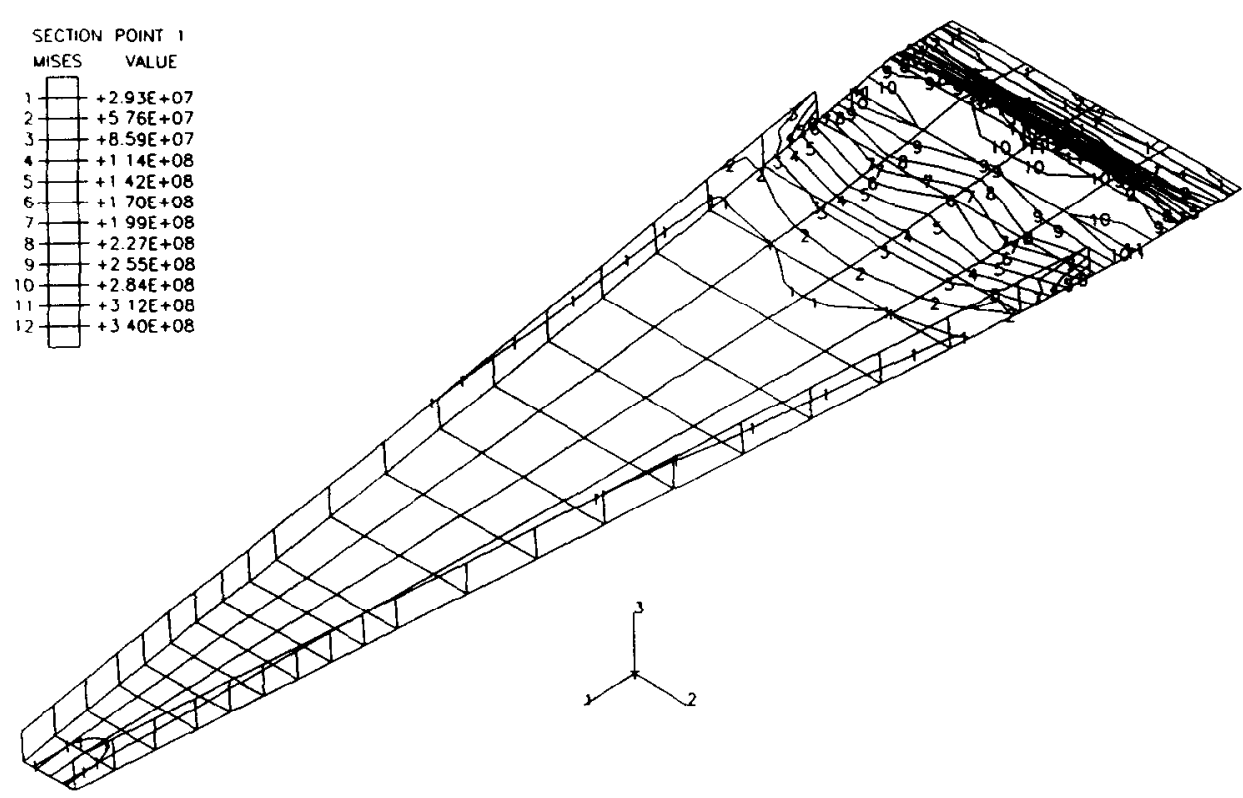

(b)

Fig. 4. (a) Loading process. (b) pre-stress distribution in the loaded state of the suspension assembly.

likely that both these modes are excited simultaneously.

The above two objectives are discrepant with each other. The farther the second and the third natural frequencies separate, the lower the first natural frequency would be. Multiobjective optimization techniques will compromise those objectives that conflict and produce satisfactory optimal design $[19,20]$. 
Table 1. Design parameters (mm)

\begin{tabular}{lccc}
\hline Design variable & Original & Minimum & Maximum \\
\hline$y b$ & 6.80 & 5.00 & 12.00 \\
$x d$ & 1.00 & 0.50 & 7.00 \\
$x 2$ & 1.90 & 0.10 & 13.00 \\
$w h$ & 0.65 & 0.30 & 0.80 \\
$l$ (fixed) & 24.80 & & \\
$t h$ (fixed) & 0.076 & & \\
$y d$ (fixed) & 0.600 & & \\
\hline
\end{tabular}

The multiobjective optimization problem can be stated as follows:

minimize the cost functionals

$$
\begin{gathered}
f_{1}=\frac{1}{\omega_{1}} \\
f_{2}=\frac{1}{\omega_{2}} \\
f_{3}=\frac{1}{\omega_{3}-\omega_{2}}
\end{gathered}
$$

subject to the minimum and maximum values of design variables, which are listed in Table 1 with their initial guesses.

The optimization of the suspension assembly is investigated with two techniques: goal programming and compromise programming. The principles and solution procedures have been described in Ref. [20]. The general formulations and their optimization philosophy are stated in Appendix B. These optimization algorithms are provided by MOST [21], in which the design variables, initial sizes of the suspension assembly, optimization techniques, objective functions, gradient calculation, and so on, are defined and coded in $C$ language. The ABAQUS is called internally by MOST whenever the structural analysis for eigenvalues and eigenvectors are requested. The designer can either terminate the design loop for any feasible intermediate design, or wait for the final results.

It is worth noticing that the minimum and maximum values of each objective function must be calculated independently in the domain of the design variable space, as shown in Table 2 . The values of a set of design variables that lead to a minimum value of $f_{i}^{*}$, namely the ideal solution of each cost function $f_{i}$, is not always in the feasible region. Therefore, the best compromise solution is searched over all feasible solutions by minimizing their distances from ideal solutions; thus the compromise between objective functions yields an optimum.

\section{ABAQUS/MOST INTERFACE}

The optimization is performed by the multiobjective optimization program MOST and the finite element analysis program ABAQUS. An interface between these two programs is developed on a workstation under the UNIX operating system. The sensitivity information is obtained to calculate gradients numerically by finite differences. Not a single source code has to be written except that system calls and file management are organized in the interface program, namely a programming -free interface. The optimization of the suspension assembly is then investigated with goal programming and compromise programming techniques provided by MOST. The final design is decided by the decision maker through additional engineering knowledge and experience.

\section{1. $M O S T$}

MOST is a multifunctional optimization system tool written in $\mathrm{C}$ language. It provides three multiobjective optimizers, the compromise programming, goal programming and the surrogate worth trade-off method for decision makers to find the best compromise solution. MOST can be used in the batch or interactive mode of computation. Only minimal input data are required for solving problems, and algorithmic parameters are automatically selected in the program. In addition, the designer can deal with continuous and noncontinuous variables simultaneously.

The computational flow of the gradient-based optimization algorithm in MOST is composed of the following steps:

(1) Give a current point in the design space, and evaluate the objective and constraint functions.

(2) Calculate gradients of the objective and constraint functions with respect to each design variable. The user has an option either to program his selfdefined gradients or to automatically calculate gradients using forward, backward or central finite differences.

(3) Based on the values of functions and gradients, determine a descent direction that minimizes the descent function. Along the direction, determine a step size to shift the design point to a new one, and evaluate the new objective and constraint functions.

(4) If any constraint is violated or convergence test is not satisfied, adjust the current point and repeat steps (1)-(4) until a final solution is obtained.

Table 2. Minimum and maximum values of objective functions

\begin{tabular}{lcc}
\hline Objective function & Minimum value $\left(f_{i}^{*}\right)$ & Maximum value $\left(f_{i \text { max }}\right)$ \\
\hline$f_{1}$ & $3.5945 \times 10^{-4}$ & $1.1643 \times 10^{-3}$ \\
$f_{2}$ & $2.5669 \times 10^{-4}$ & $8.2026 \times 10^{-4}$ \\
$f_{3}$ & $2.2668 \times 10^{-4}$ & $1.9534 \times 10^{-3}$ \\
\hline
\end{tabular}




\subsection{ABAQUS}

The software package ABAQUS is designed to provide for advanced structure analyses. The most important features are applications to either linear analysis of very large linear models, or large models with highly nonlinear response. The theoretical formulation is based on the finite element stiffness method, with some 'hybrid' (mixed stress-displacement variable) formulations included as necessary. One of the most attractive features is 'step', a portion of the analysis histury, such as a dynamic event, a stage in manufacturing process, or modal analysis under a preloading force. ABAQUS then automatically selects increments to model the step. This approach is extremely effective for nonlinear problems, since structural dynamical responses may change drastically during an analysis step.

\subsection{MOST-based interface structure}

The interface between the finite element program ABAQUS and the optimizer MOST is based on the MOST programming structure under the UNIX operating system. In the proposed interface architecture, MOST acts as a master program which internally invokes the execution of command files to perform the finite element analysis in ABAQUS. The interface program can be carried out between a workstation and any remote terminal through the connection of the network. The UNIX operating system coded in C language makes this architecture possible and efficient because:

(1) the master program, which acts like an interactive file organizer, can sleep or suspend with no action while it is forking a child process;

(2) data written in binary or ascii code can be transferred through the network;

(3) the gradient calculations based on finite difference approaches are independent for each iteration, without the calculation of element stiffness and mass matrices.

The computational flow of the optimization and structural analysis is shown in Fig. 5.

\section{SENSITIVITY TO DESIGN VARIABLES}

In most of the efficient optimization methods, the design sensitivity analysis is required to determine the derivatives of the objective functions with respect to the parameters of interest. In this paper we do not obtain the sensitivity derivatives explicitly from equations. Instead, the sensitivities of natural frequencies of the suspension assembly to the design variables are numerically investigated. The purposes of plotting the sensitivity analysis are:

(1) the designer may want to discard those design variables that are least sensitive to the natural frequencies:

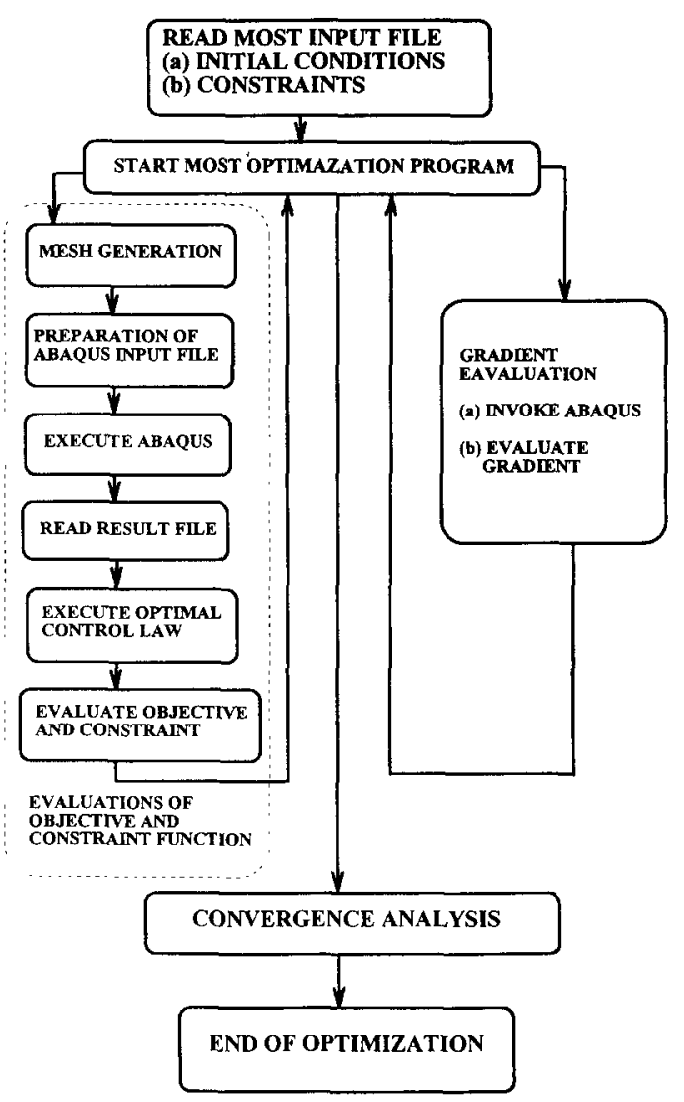

Fig. 5. MOST-ABAQIJS interface flow diagram.

(2) the designer may keep constants of those design variables to which the sensitivities are linear, or monotonic functions; and

(3) only the design variables that arc not involved with the above two cases are retained for the subsequent optimal design.

In this way the number of the design variables can be kept minimal to expedite the design optimization of structures. On the other hand, decision makers can make a proper shift or modification of the final solutions according to the linearity of sensitivity with respect to a certain structure parameter.

In Figs 6-11 the sensitivity curves of the first three natural frequencics are displayed with respect to a single design variable, while other design variables are fixed as the original values of the suspension assembly. We found that in Fig. 6 the first two natural frequencies decrease as the width of the base of the suspension $y b$ increases, but the third natural frequency has a maximum value. Figure 7 shows that the first and third natural frequencies decreases as $x d$, the length of the suspension base attached on the mounting block, increases. The second natural frequency has a maximum value as $x d$ is around $10 \mathrm{~mm}$ at which, however, the difference between the second and the third natural frequencies seems to be a minimum. There should have been a compromise 


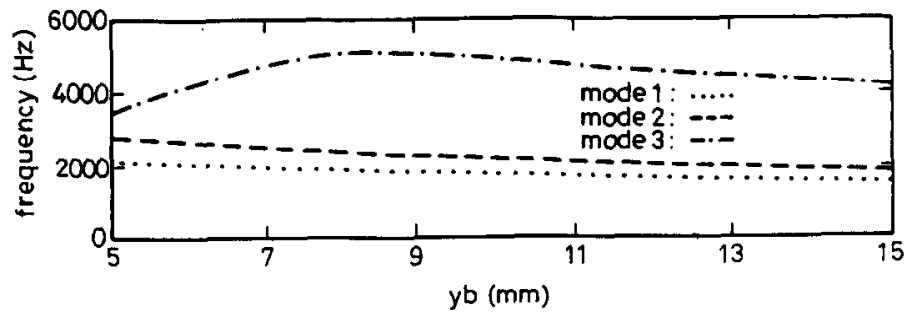

Fig. 6. Sensitivities of natural frequencies with respect to $y b$.

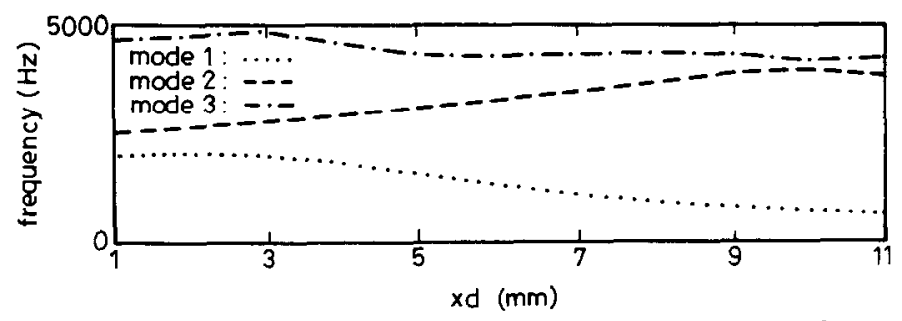

Fig. 7. Sensitivities of natural frequencies with respect to $x d$.

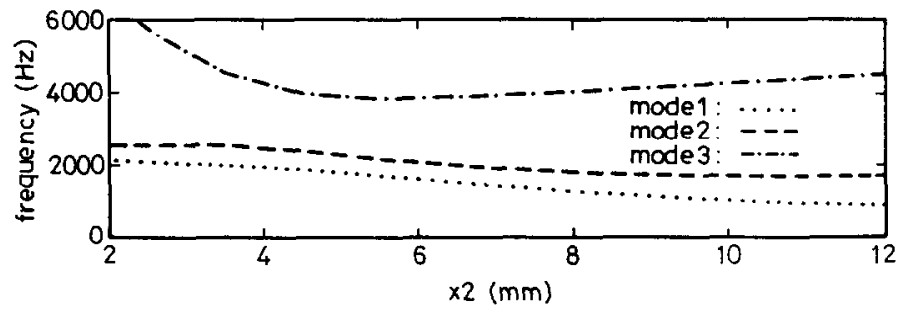

Fig. 8. Sensitivities of natural frequencies with respect to $x 2$.

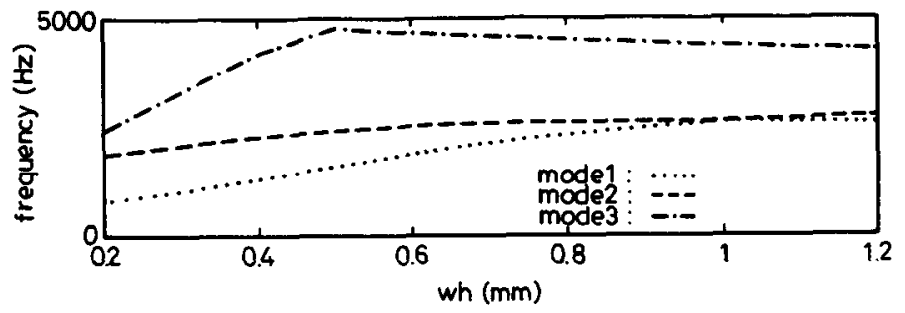

Fig. 9. Sensitivities of natural frequencies with respect to wh.

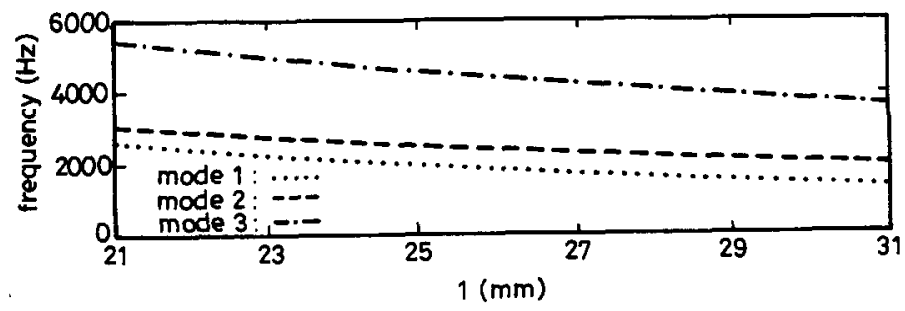

Fig. 10. Sensitivities of natural frequencies with respect to $l$. 


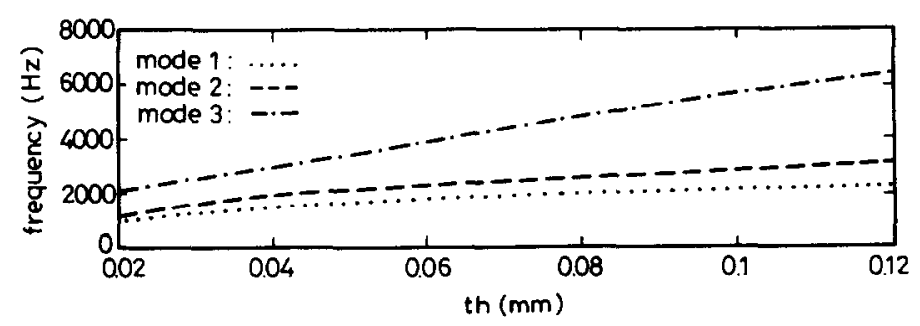

Fig. 11. Sensitivities of natural frequencies with respect to $t h$.

between the design objectives. In Fig. 8, the natural frequencies tend to decrease as $x 2$, the distance between the bending corner and the suspension flaps, increases, while the sensitivity curve of the third natural frequency has a minimum. The maximum difference of the second and the third natural frequencies seems to happen in the region where $x 2$ is less than $3 \mathrm{~mm}$. Figure 9 shows that the first two natural frequencies are increasing smoothly as the height of the suspension flaps $w^{h}$ is increasing, whereas the third natural frequency reaches a maximum as $w h$ is around $0.5 \mathrm{~mm}$ and separates the most from the second natural frequency.

From a structural dynamics point of vicw, it is essential that the longer the suspension is, the smaller its natural frequencies are; and the thicker the suspension beam is, the larger its natural frequencies are. These facts are shown clearly in Figs 10 and 11. It is obvious that the natural frequencies of the suspension assembly vary nonlinearly with the design variables $y b, x d, x 2$, and $w h$, nevertheless the sensitivity curves are approximately linear with variables $l$ and $t h$. For the subsequent optimal design, $l$ and $t h$ are not selected as design variables since their corresponding sensitivity curves are monotonic. Besides, the length and thickness of the suspension beam are usually determined under the restrictions on the size and space of the hard disk drives for manufacturing.

\section{OPTIMIZATION RESULTS}

\subsection{Preloading}

The optimization process is performed with the suspension assembly in its loaded status. The first six natural frequencies of the original suspension in the unloaded and loaded positions are compared in Table 3. Due to the preload induced pre-strain and prestress in the suspension beam, the natural frequencies of the first bending mode and the first torsional mode become smaller than those of unloaded beam. This preloading process is accomplished by the feature of 'step' defined as a portion of analysis history in ABAQUS.

\subsection{Optimal shape design}

The optimal design results of the suspension assembly by the use of goal and compromise programmings for $\beta, \gamma=1$ and 2 are listed in Tables 4 and 5, respectively. The optimal shapes in the finite element mesh for the cases of $\beta=1$ and $\gamma=1$ are illustrated in Fig. 12.

We can conclude the following:

(1) The flap height can be built with its maximum allowable value, which is $0.8 \mathrm{~mm}$ in our design. The designer must also consider the vertical dimensions occupying the space between disks. This spacing is calculated by summing slider height, suspension offset, flap height, loading clearance, baseplate height and arm thickness.

(2) The final results on raising natural frequencies are very satisfactory. Without too much change from the original shape of the suspension, the first and second natural frequencies are raised over $400-500 \mathrm{~Hz}$. Also, the difference between the second and third natural frequencies increases over $1000-2000 \mathrm{~Hz}$.

(3) The final value of each objective function, as shown in Tables 4 and 5 , is larger than its minimum value and less than its maximum value, as shown in Table 2. In other words, the nondominated solution, that happens when a set of design variables make all the objectives minimum, does not exist. Instead, an optimal solution is obtained by making a compromise between objective functions with each other.

(4) There are no apparent facts that reveal which design variables dominate the variation of

Table 3. Natural frequencies of suspension assembly

\begin{tabular}{lcccc}
\hline & \multicolumn{2}{c}{ Unloaded } & \multicolumn{2}{c}{ Loaded } \\
Mode & Frequency $(\mathrm{Hz})$ & Type & Frequency (Hz) & Type \\
\hline 1 & 2075 & bending & 2057 & bending \\
2 & 2713 & torsional & 2608 & torsional \\
3 & 5654 & torsional & 4645 & torsional \\
4 & 5852 & bending & 5779 & bending \\
5 & 7022 & torsional & 7228 & torsional \\
6 & 10942 & bending & 11057 & bending \\
\hline
\end{tabular}


Table 4. Design results with goal programming

\begin{tabular}{lcc}
\hline & $\beta=1$ & $\beta=2$ \\
\hline Design variable $(\mathrm{mm})$ & & \\
$y b$ & 7.6683 & 7.1136 \\
$x d$ & 1.7676 & 2.2518 \\
$x 2$ & 0.4335 & 0.5181 \\
$w h$ & 0.8000 & 0.8000 \\
& & \\
Objectives & & \\
$f_{1}$ & $3.9489 \times 10^{-4}$ & $3.9367 \times 10^{-4}$ \\
$f_{2}$ & $3.8020 \times 10^{-4}$ & $3.6245 \times 10^{-4}$ \\
$f_{3}$ & $2.5802 \times 10^{-4}$ & $2.8014 \times 10^{-4}$ \\
Errors & & \\
$d_{1}^{+}$ & & \\
$d_{2}^{+}$ & $1.3544 \times 10^{-4}$ & $0.3422 \times 10^{-4}$ \\
$d_{3}^{+}$ & $1.2351 \times 10^{-4}$ & $1.0576 \times 10^{-4}$ \\
& $0.3134 \times 10^{-4}$ & $0.5346 \times 10^{-4}$ \\
Frequencies $(\mathrm{Hz})$ & & \\
$\omega_{1}$ & & \\
$\omega_{2}$ & 2532 & 2540 \\
$\omega_{3}$ & 2630 & 2759 \\
\hline
\end{tabular}

natural frequencies. To achieve multiple objectives, minimizing their values, different optimization techniques result in different final solutions. Decision makers must choose the best or most proper design with additional engineering knowledge and experience.

\subsection{Decision making}

The design objective is to raise natural frequencies of the suspension assembly so that it will not be excited easily by undesirable disturbances. This motivates the designer to investigate frequency responses of the optimal suspension shape under the action of disturbances. Assume that the major disturbance comes from the turbulence flow between disks and exerts a vertical force perpendicular to the horizontal surface of the suspension. One may take the centroid of the suspension beam as a disturbance input point where the resultant force concentrates, and choose a bottom corner where the head coil locates as the displacement output point. In such cases, the symmetrical torsional modes are uncontrollable. The transfer function of the system with the force input and displacement output can be easily derived by modal equations from the finite element analysis of the resulting optimal suspension assembly.

Three transfer functions are formulated by selecting the first six modes of the corresponding design of the suspension assembly of the original dimensions, goal programming and compromise programming, respectively. Note that modes 2, 3 and 5 are torsional modes, symmetric and hence uncontrollable, and the modal magnitude of mode 6 is much less than those of other bending modes, therefore only two bending modes are present in the Bode plots as shown in Fig. 13. The dashed curve represents the Bode plot of the original suspension system, which has the highest d.c. gain and resonant peak. Both the d.c. gain and resonant peak in the Bode plot of the suspension with compromise programming $(\gamma=1)$ design reduce about $5 \mathrm{~dB}$, while those with goal programming $(\beta=1)$ design reducc about $9 \mathrm{~dB}$. From the frequency response point of view, the decision maker may choose the final design with goal programming techniques.

In the frequency response analysis, the modal damping ratio 0.07 is chosen for each mode. For further reduction of the resonant peak, the designer can use a passive control device by adding a layer of damping material to the suspension [22], or use active control methods to suppress unwanted vibrations during the disk operation.

\section{SUMMARY AND CONCLUSIONS}

The optimization of the suspension assembly of hard disk drives, by treating the natural frequencies as objective functions, has been presented. The design objective is to maximize natural frequencies of the suspension assembly in order not to be easily excited by undesirable disturbances. The design variables are geometrical sizes of the suspension assembly, and are reduced to four by the sensitivity analysis. It is investigated that the natural frequencies are monotonic functions of the thickness and length of the suspension. Therefore, the design variables that are maintained for optimizations are the width of the base of the suspension, the length of the suspension base attached on the mounting block, the distance between the bending corner and the suspension flaps, and the height of the suspension flaps. It is interesting to find out that the sensitivities of natural frequencies with respect to the above four design variables are nonlinear. The compromise between these four design variables results in an optimal solution that satisfies the design objectives. More design variables, such as the size and shape of the slider, flexure and actuating arm, can be chosen subject to manufacturing limitations, physical constraints, or design efficiency.

The objectives of the optimization are multifunctions which consist of the reciprocals of the first

Table 5. Design results with compromise programming $(\alpha=1)$

\begin{tabular}{lcc}
\hline & $\gamma=1$ & $\gamma=2$ \\
\hline Design variable (mm) & & \\
$y b$ & 6.8513 & 6.3979 \\
$x d$ & 2.4359 & 2.9607 \\
$x 2$ & 0.3533 & 0.4663 \\
$w h$ & 0.8000 & 0.8000 \\
& & \\
Objectives & & \\
$f_{1}$ & $3.8967 \times 10^{-4}$ & $4.0530 \times 10^{-4}$ \\
$f_{2}$ & $3.5747 \times 10^{-4}$ & $3.4127 \times 10^{-4}$ \\
$f_{3}$ & $2.9920 \times 10^{-4}$ & $3.4601 \times 10^{-4}$ \\
Frequencies (Hz) & & \\
$\omega_{1}$ & & \\
$\omega_{2}$ & 2566 & 2467 \\
$\omega_{3}$ & 2797 & 2930 \\
\hline
\end{tabular}


natural frequency, the second natural frequency and the difference between the second and third natural frequencies, subject to the side constraints on the design variables. The development of an interface between the finite element program ABAQUS and the multifunctional optimization program MOST on a workstation under the UNIX operating system is demonstrated. Both programs have powerful

(a)

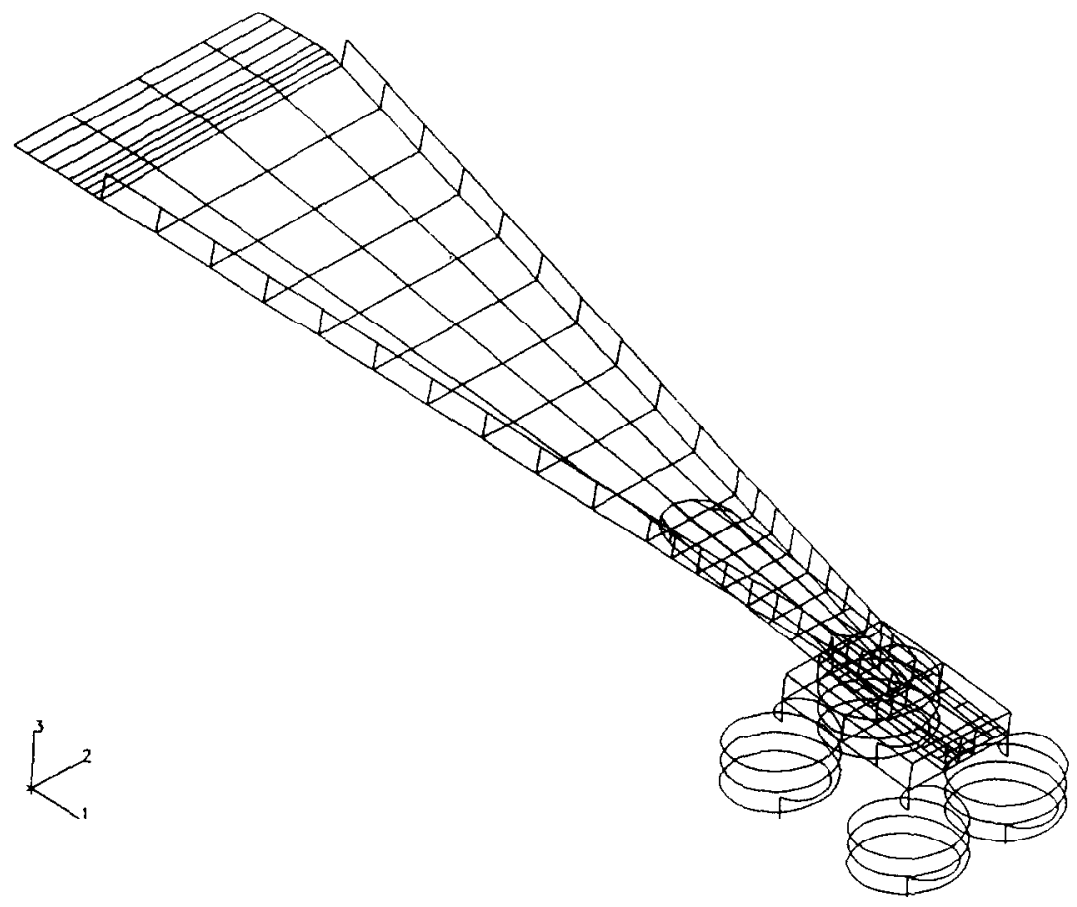

(b)

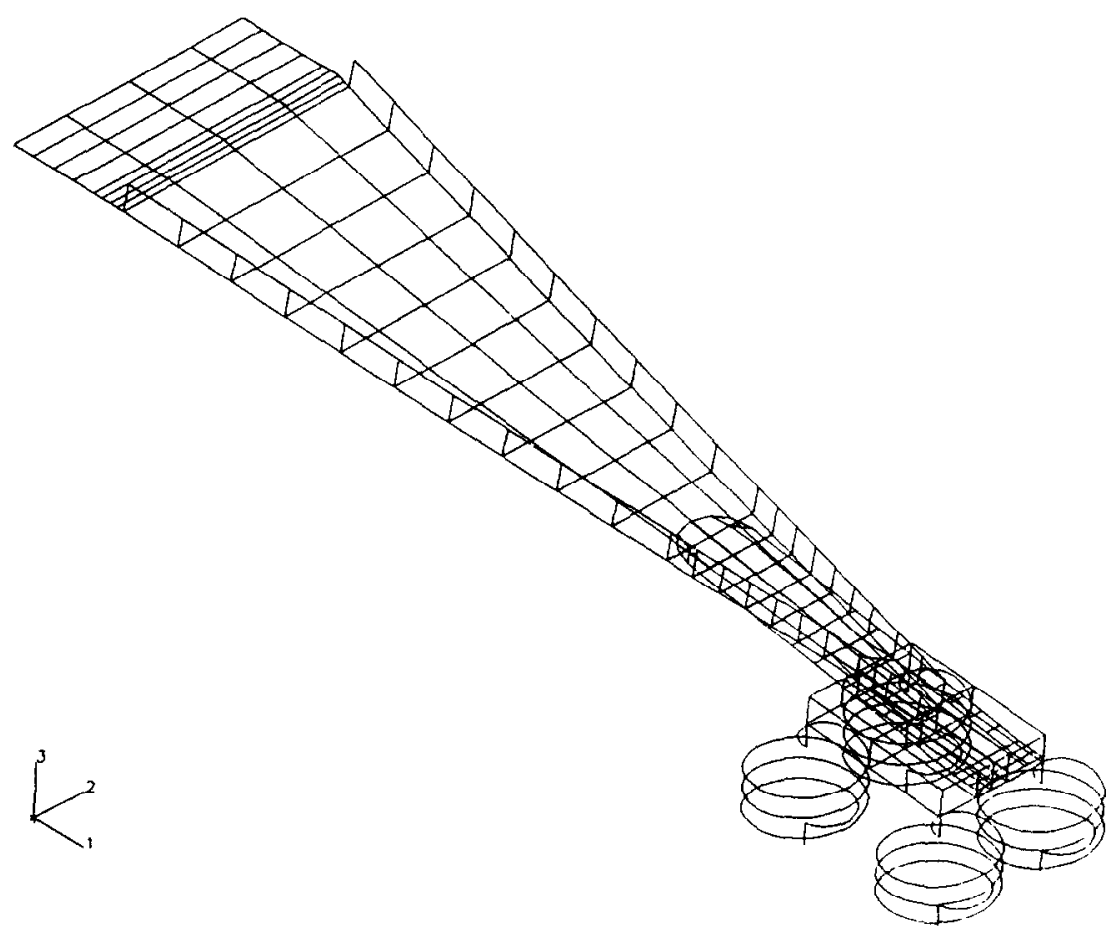

Fig. 12. Optimal design results. (a) Goal programming $(\beta=1)$. (b) Compromise programming $(\gamma=1)$. 


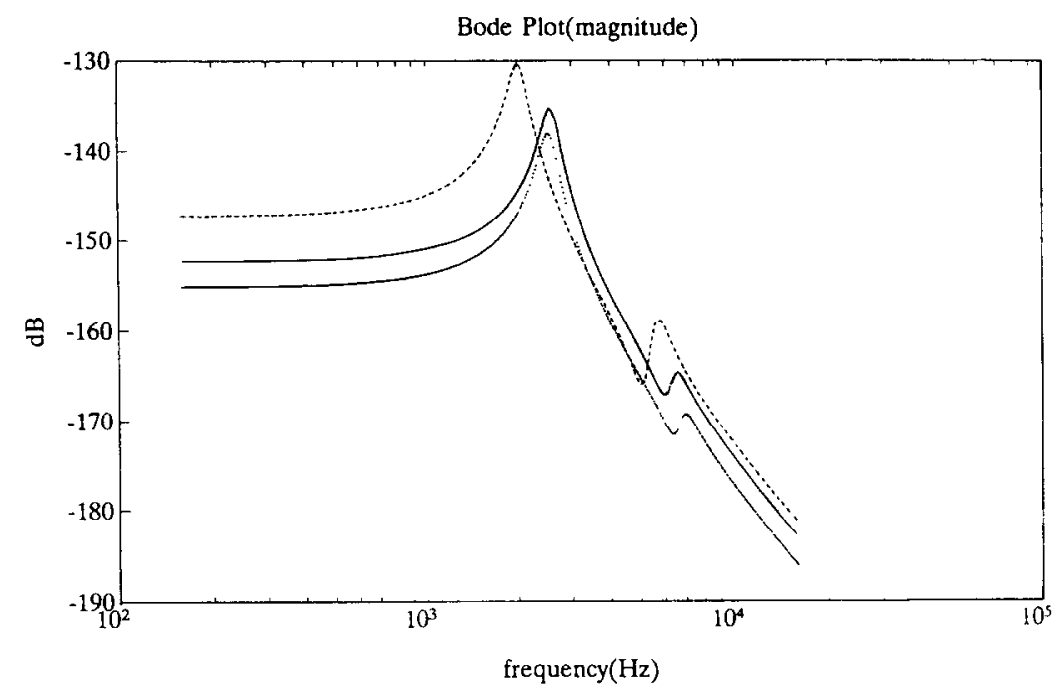

Fig. 13. Frequency response of suspension assemblies (dashed curve: original system, solid curve compromise programming, dotted curve: goal programming).

capabilities, respectively, on analysis and optimization. Thus not a single source code has to be written except that system calls and file management are organized in the interface program, namely, a programming-free interface. The main purpose of this interface is to make the most of existing well-developed software packages on available computer facilities. Besides, the designer can focus on his design objectives and decision making without getting involved with tedious programming work on coding and debugging.

The optimization of the suspension assembly is investigated with two multiobjective optimization techniques-goal programming and compromise programming. Different design philosophies yield various optimal design results. Decision makers, therefore, have to choose the best or most proper design based on their engineering knowledge and experience, such as frequency response, transient response and material characteristics, and so on.

Acknowledgements - This research was supported by the National Science Council under Contract no. NSC 81-0401E-002-552.

\section{REFERENCES}

1. S. Yoneoka, T. Owe, K. Aruga, T. Yamada and M. Takahashi, Dynamics of inline flying-head assemblies. IEEE Trans. Magn. 25, 3716-3718 (1989).

2. R. D. Cormia and D. Mann, Contact recording on rigid disks: materials and materials analysis. Solid State Technol. March, 59-61 (1992).

3. K. Tanaka, Y. Takeuchi, S. Terashima, T. Odaka and Y. Saitoh, Measurements of transient motion of magnetic disk slider. IEEE Trans. Magn. MAG-20, 924-926 (1984).

4. J. Naruse, M. Tsutsumi, T. Tamura, Y. Hirano, T. Hayama and O. Matsushita, Design of a large capacity disk drive with two actuators. IEEE Trans. Magn. 19, 1695-1697 (1983).

5. Y. Yamaguchi, A. A. Talukder, T. Shibuya and M. Tokuyama, Air flow around a magnetic-head-slider suspension and its effect on slider flying-height fluctuation. IEEE Trans. Magn. 26, 2430-2432 (1990).

6. M. Takuyama, Y. Yamaguchi, S. Miyata and C. Kato, Numerical analysis of flying-height fluctuation and positioning error of magnetic head due to flow induced by disk rotation. IEEE Trans. Magn. 27, 5139-5141 (1991).

7. H. Hanselmann and W. Moritz, High-bandwidth control of the head-positioning mechanism in a Winchester disk drive. IEEE Control Syst. Mag. October, 15-19 (1987).

8. Y. Mizoshita, K. Aruga and T. Yamada, Dynamic characteristics of a magnetic head slider. IEEE Trans. Magn. 21, 1509-1511 (1985).

9. J. Felix and G. N. Vanderplaats, Configuration optimization of trusses subject to strength, displacement and frequency constraints. Trans. ASME, J. Mech. Transmiss. Automat. Des. 109, 233-241 (1987).

10. A. Chattopadhyay and J. L. Walsh, Minimum weight design of rotorcraft blades with multiple frequency and stress constraints. AIAA J. 28, 565-567 (1990).

11. O. G. McGee and K. F. Phan, A robust optimality criterion procedure for cross-sectional optimization of frame structures with multiple frequency limits. Comput. Struct. 38, 485-500 (1991).

12. R. Grandhi, Structural optimization with frequency constraints-a review. AIAA J. 31, 2296-2303 (1993).

13. R. A. Canfield, High-quality approximation of eigenvalues in structural optimization. AIAA J. 28, 1116-1122 (1990).

14. M. P. Bendsoc, N. Olhoff and J. E. Taylor, A variational formulation for multicriteria structural optimization. J. Struct. Mech. 11, 523-544 (1983-84).

15. W. Szyszkowski, Multimodal optimality criterion for maximum fundamental frequency of free vibrations. Comput. Struct. 41, 909-916 (1991).

16. W. Szyszkowski and J. M. King, Optimization of frequencies spectrum in vibrations of flexible structures. AIAA J. 31, 2163-2168 (1993).

17. O. J. Ruiz and D. B. Bogy, A numerical simulation of the hard-disk assembly in magnetic hard disk files: Part I-component models. Trans. ASME J. Tribol. 112, 593-602 (1990).

18. L. J. Pan, The control in fiying height of slider in hard disk. Master thesis, Department of Mechanical Engineering. National Tiawan University, Republic of China (1993). 
19. F. Szidarovszky, M. E. Gershon and L. Duckstein, Techniques for Multiobjective Decision Making in System Management. Elsevier, New York (1986).

20. C. H. Tseng, T. W. Lu and L. W. Wang, Multiobjective optimization with non-continuous design variables. J. Chinese Soc. Mech. Engng 13, 547-560 (1992).

21. C. H. Tseng, W. C. Liao and T. C. Yang, MOST User's Manual Version 1.1, Technical Report no. AODL-9301, National Chiau-Tung University, Taiwan (1993).

22. A. D. Nashif, D. I. G. Jones and J. P. Henderson, Vibration Damping. Wiley, New York (1985).

23. G. W. Evans, An overview of techniques for solving multiobjective mathematical programs. Mgmt Sci. 30, 1268-1282 (1984)

24. M. Zeleny, Multiple Criteria Decision Making. McGraw-Hill, New York (1982).

APPENDIX A

ORIGINAL DIMENSIONS OF SUSPENSION BEAM, FLEXURE AND SLIDER

A. Suspension beam and flexure (unit: $\mathrm{mm}$ and degrees)

\begin{tabular}{lrrlrrrr}
\hline XL & 24.8 & X0 & 1.0 & X1 & 1.5 & X2 & 3.4 \\
YL & 6.8 & TH1 & 0.076 & ZL & 0.65 & $\theta$ & 10.0 \\
A1 & 9.8 & A2 & 1.9 & A3 & 0.6 & A4 & 2.45 \\
A5 & 4.8 & A6 & 5.2 & A7 & 0.55 & A8 & 0.7 \\
A9 & 0.9 & A10 & 1.1 & A11 & 1.1 & A12 & 0.95 \\
A13 & 0.15 & A14 & 3.005 & A15 & 4.05 & A16 & 0.75 \\
A17 & 2.42 & A18 & 0.1 & A19 & 2.025 & A20 & 0.44 \\
A21 & 2.163 & A22 & 0.051 & TH2 & 0.051 & $\theta_{1}$ & 15.0 \\
\hline
\end{tabular}

$E=2.0601 \times 10^{11} \mathrm{~N} \mathrm{~m}^{-2}$ (Young's modulus)

$d=7.8 \times 10^{3} \mathrm{~kg} \mathrm{~m}^{-3}$ (density)

$\mu=0.33$ (Poisson ratio)

B. Slider (unit: $\mathrm{mm}$ )

\begin{tabular}{llcccc}
\hline XL & 4.064 & XT & 0.381 & XG & 2.142 \\
YL & 0.686 & YGYL & 1.601 & HT & $0.5542 \times 10^{-2}$ \\
XF0 & 2.170 & & & & \\
\hline
\end{tabular}

$m=0.7207 \times 10^{-10} \mathrm{~kg} \mathrm{~s}^{2} \mathrm{~m}^{-1}$ (mass)

$I_{\mathrm{p}}=0.1457 \times 10^{-10} \mathrm{~kg} \mathrm{f} \mathrm{s}{ }^{2} \mathrm{~m}$ (pitch moment of inertia)

$I_{\mathrm{r}}=0.7562 \times 10^{-11} \mathrm{~kg} \mathrm{f} \mathrm{s}{ }^{2} \mathrm{~m}$ (roll moment of inertia)

$I_{4}=0.1457 \times 10^{-10} \mathrm{~kg} \mathrm{fs} \mathrm{s}^{2} \mathrm{~m}$ (skew moment of inertia)

\section{APPENDIX B}

\section{MULTIOBJECTIVE OPTIMIZATION ALGORITHMS} IN MOST

\section{Goal programming}

The ohjective of goal programming is to minimize the errors between the optimal solution and the ideal solution in the objective function space. The errors, namely the under-achievement and over-achievement of the $i$ th objective function $f_{i}(x)$, are defined, respectively, by

$$
\begin{aligned}
& d_{i}^{+}=0.5\left[\left|f_{i}(x)-T_{i}^{*}\right|+\left(f_{i}(x)-T_{i}^{*}\right)\right] \\
& d_{i}^{-}=0.5\left[\left|f_{i}(x)-T_{i}^{*}\right|-\left(f_{i}(x)-T_{i}^{*}\right)\right],
\end{aligned}
$$

where $T^{*}$ represents the target or goal set by the decision maker for the $i$ th objective function. Therefore, the general formulation for nonlinear optimization problems can be stated as [23]

$$
\min F(x)=\left\{\sum_{i=1}^{q}\left(d_{i}^{+}+d_{i}^{-}\right)^{\beta}\right\}^{1 / \beta} ; \beta \geqslant 1
$$

subject to the constraints

$$
\begin{array}{r}
h_{j}(x)=0 ; j=1,2, \ldots, p \\
g_{\jmath}(x) \leqslant 0 ; j=1,2, \ldots, m \\
T_{i}(x)=f_{i}(x)-d_{i}^{+}+d_{i}^{-} ; i=1,2, \ldots, q \\
d_{1}^{+}, d_{1}^{-} \geqslant 0 ; i=1,2, \ldots q .
\end{array}
$$

in which the value of $\beta$ is based on the utility function chosen by the decision maker, and it is possible to obtain nondominated solutions by varying the values of $\beta$ and $T$,

\section{Compromise programming}

The compromise programming [24] is to minimize the distance between the ideal solution and the optimal solution that is so-called the compromise solution. The distance measure used in compromise programming to evaluate how close the set of nondominated points come to the ideal point is the family of $L_{\eta}$ matrices defined as

$$
\min L_{\gamma}=\left\{\sum_{i=1}^{4} \alpha_{i}^{\prime \prime}\left|\frac{f_{i}(x)-f_{i}^{*}}{f_{t, \max }-f_{i}^{*}}\right|^{\prime}\right\}^{1 / ;}, \quad 1 \leqslant \gamma<\infty
$$

subject to $x \in X$, in which $\alpha_{1}$ are weights, $f_{1}^{*}$ and $f_{l, \max }$ are, respectively, the minimum value and the worst value of the $i$ th objective function, $f_{i}(x)$, is the value of implementing the design variable $x$ with respect to the $i$ th objective, and $X$ is the feasible design space.

In the case of $\gamma=1$, all deviations from $f_{1}^{*}$ are taken into consideration in proportion to their magnitudes. For $2 \leqslant \gamma<\infty$, the larger the deviation, the larger the weight in $L_{\gamma}$. In the limiting case of $\gamma=\infty$ the largest deviation from the minimum objectives only is considered. 